Quim. Nova, Vol. 31, No. 3, 556-561, 2008

\title{
INFLUÊNCIA DE MÉTODOS DE DIGESTÃo E MASSA DE AMOSTRA NA RECUPERAÇÃO DE NUTRIENTES EM RESÍDUOS ORGÂNICOS
}

\author{
Leônidas Carrijo Azevedo Melo* e Carlos Alberto Silva \\ Departamento de Ciência do Solo, Universidade Federal de Lavras, CP 3037, 37200-000 Lavras - MG, Brasil
}

Recebido em 14/3/07; aceito em 27/9/07; publicado na web em 10/3/08

\begin{abstract}
INFLUENCE OF DIGESTION METHOD AND SAMPLE MASS ON THE RECOVERY OF NUTRIENTS IN ORGANIC RESIDUES. The influence of nitric-perchloric, aqua regia, dry ashing and microwave digestion methods, in combination with 100 and $200 \mathrm{mg}$ of sample, on the characterization and recovery of nutrients in samples of sludge sewage, poultry, swine, quail and bovine manures, organic compost, organic substrate and humic material were studied. Nitric-perchloric digestion with $200 \mathrm{mg}$ samples recovered the higher nutrient contents. The nitric-perchloric method recovered also low levels of K. Dry ashing caused S volatilization and microwave digestion produced dark color extracts and this impaired $\mathrm{S}$ determination. Aqua regia recovered the lowest contents of nutrients in the organic residues evaluated.
\end{abstract}

Keywords: acid digestion; recovery index; nutrient content.

\section{INTRODUÇÃO}

O uso eficiente de resíduos orgânicos como fertilizantes na agricultura depende do conhecimento da composição química desses materiais. Uma das etapas mais críticas da caracterização é a digestão das amostras. Nessa fase, são utilizados sistemas fechados e abertos de digestão, com o emprego de diferentes combinações de ácidos, quase sempre com índices variáveis de recuperação dos elementos químicos presentes nos resíduos, o que se explica devido à grande variação no grau de polimerização, natureza química e presença nos resíduos orgânicos de moléculas mais e menos resistentes ao ataque químico. ${ }^{1}$

Em estudo comparativo entre a digestão em recipiente aberto com $\mathrm{HNO}_{3}$ concentrado e a digestão em microondas com $\mathrm{HNO}_{3}+$ $\mathrm{H}_{2} \mathrm{O}_{2}$, para a determinação de metais em amostras de cereais e troncos de coníferas, Rodushkin et al. ${ }^{2}$ verificaram que ambos os procedimentos garantem uma preparação rápida de várias amostras, mas a digestão em recipiente aberto tem a vantagem do baixo custo de equipamento e menor necessidade de pré-tratamento de amostras. Zheljazkov e Warman ${ }^{3}$ compararam os métodos de digestão com ácido nítrico, mistura nítrico-perclórica e em mufla, para determinar 17 elementos químicos em seis compostos oriundos do Canadá. Foi verificado que o ácido nítrico proporcionou a maior recuperação de As, Mo, Ni e Se e que a digestão com mistura nítrico-perclórica propiciou a maior recuperação de Co e a mufla, de Mn.

Em geral, as técnicas convencionais de dissolução por via úmida consomem muito tempo e algumas marchas analíticas são potencialmente suscetíveis à contaminação e à perda de elementos químicos por volatilização. A queima das amostras em mufla pode fazer com que alguns elementos químicos sejam perdidos por volatilização ou adsorvidos na parede do forno. ${ }^{4}$ A digestão assistida por microondas apresenta vantagens, pois reduz o tempo de preparação, bem como os riscos associados aos métodos clássicos, tais como contaminação e perda dos elementos químicos voláteis. Por outro lado, segundo Sastre, ${ }^{5}$ os sistemas abertos de digestão permitem a análise simultânea de um número maior de amostras, facilitam a evaporação de ácido, a secagem dos extratos digeridos

*e-mail: leonidasmelobr@yahoo.com.br e reduzem os problemas analíticos em etapas subseqüentes à digestão. Uma demanda recorrente está ligada à avaliação dos índices de recuperação de elementos químicos, proporcionados pelas diferentes combinações de mistura de ácidos com sistemas de aquecimentos das amostras na digestão.

A obtenção de resultados confiáveis no laboratório inicia-se com a adequada "abertura" das amostras para as posteriores determinações analíticas. ${ }^{6}$ Em muitos casos, recomenda-se realizar uma pré-digestão das amostras, geralmente com peróxido de hidrogênio $\left(\mathrm{H}_{2} \mathrm{O}_{2}\right)$ ou aquecimento a $550{ }^{\circ} \mathrm{C} .{ }^{7}$ A massa de amostra utilizada exerce um papel importante para a avaliação da homogeneidade dos materiais e para a decomposição completa da matéria orgânica durante o processo de digestão. Em estudo sobre a digestão de resíduos orgânicos diversos, Boeira et al. ${ }^{8}$ testaram diferentes massas de amostra (50, 100 e $200 \mathrm{mg}$ ) e métodos de digestão (nítricoperclórica; água-régia e ácido sulfúrico $+\mathrm{H}_{2} \mathrm{O}_{2}$ ) e verificaram que a massa de $200 \mathrm{mg}$ com ácido sulfúrico $+\mathrm{H}_{2} \mathrm{O}_{2}$ recuperou maiores teores de $\mathrm{Ca}, \mathrm{Mg}$ e $\mathrm{K}$. Com o objetivo de dimensionar o tamanho ideal da amostra para digestão, Alkanani et al. ${ }^{9}$ testaram, do mesmo modo, diferentes massas $(200,400$ e $800 \mathrm{mg})$ de material certificado de leite em pó (A-11 milk powder), em método de digestão em que foi utilizado o microondas para a determinação dos teores de diversos elementos químicos. Estes autores verificaram que o acréscimo da massa de amostra não aumentou o grau de recuperação dos elementos químicos avaliados.

Estudos anteriores estabeleceram bons procedimentos para determinação de elementos químicos em amostras de solos e sedimentos, pelo uso de ácidos inorgânicos concentrados e altas temperaturas. ${ }^{10}$ Entretanto, pouco se conhece sobre a recuperação relativa de elementos a partir de compostos orgânicos por vários métodos de digestão. ${ }^{3}$ Daí, a importância de se testar diferentes metodologias em sistemas fechados e abertos, para uma grande variedade de matrizes orgânicas, no sentido de se caracterizar com mais eficiência esses materiais que são largamente empregados na adubação das lavouras.

Com este estudo objetivou-se avaliar os métodos de digestão e as massas de amostras que proporcionam maior eficiência de recuperação dos teores de nutrientes presentes em resíduos orgânicos de origens diversas. 


\section{PARTE EXPERIMENTAL}

O experimento foi conduzido no Departamento de Ciência do Solo da Universidade Federal de Lavras, e as análises realizadas no Laboratório de Estudo da Matéria Orgânica do Solo (Lemos) e no Laboratório de Pedologia e Geoquímica Ambiental. Os materiais orgânicos foram coletados em municípios do sul de Minas Gerais. Foram analisadas amostras de lodos de esgoto (1 e 2), esterco de aves, suíno, codorna e bovino, composto orgânico, além de substrato orgânico e material húmico comerciais. Após a coleta, as amostras foram secas $\left(70{ }^{\circ} \mathrm{C}\right)$, moídas em moinho tipo Wiley e peneiradas $(0,25 \mathrm{~mm})$. A caracterização físico-química dos resíduos orgânicos estudados pode ser observada na Tabela 1 .

Os tratamentos avaliados consistiram da combinação de quatro procedimentos de digestão com duas massas (100 e $200 \mathrm{mg}$ ) de amostras, com quatro repetições, totalizando 32 unidades experimentais. Essas massas foram escolhidas com base em testes realizados previamente para a determinação do tamanho mínimo de amostra que não apresentasse problemas com a digestão, tal como o transbordamento nos tubos de digestão e, também, para verificar a heterogeneidade dos resíduos.

\section{Métodos de digestão}

\section{Digestão nítrico-perclórica (DNP)}

Utilizou-se a metodologia descrita por Tedesco et al., ${ }^{12} \mathrm{com}$ modificações. Realizou-se a digestão em bloco digestor e foram digeridas duas massas de amostras (100 e $200 \mathrm{mg}$ ), por meio da adição de $6 \mathrm{~mL}$ de $\mathrm{HNO}_{3}$ p.a., que foi misturado à amostra na noite anterior à digestão, com a amostra permanecendo em repouso por 16 h; a seguir, iniciou-se a digestão com a elevação gradual da temperatura até $140{ }^{\circ} \mathrm{C}$. A amostra foi digerida até restar cerca de 1 $\mathrm{mL}$ de ácido. Porém, quando não houve um clareamento das amostras no tubo de digestão, adicionaram-se mais $4 \mathrm{~mL}$ de $\mathrm{HNO}_{3}$ p.a. e continuou-se o processo até a diminuição do volume de ácido $( \pm 1$ $\mathrm{mL}$ ). Em seguida, os extratos foram retirados do bloco e resfriados até a temperatura ambiente. A seguir, foram adicionados $2 \mathrm{~mL}$ de $\mathrm{HClO}_{4}$ p.a. e elevada a temperatura do bloco para $190{ }^{\circ} \mathrm{C}$, permanecendo a amostra em digestão por mais $2 \mathrm{~h}$. Ao término desse prazo, as amostras foram retiradas do bloco, resfriadas em temperatura ambiente, com o extrato digerido (cerca de $2 \mathrm{~mL}$ ) completado para $10 \mathrm{~mL}$ com água bidestilada. Em seqüência, o extrato digerido foi filtrado em membrana celulósica com $0,45 \mu \mathrm{m}$ de diâmetro e transferido para frasco tipo Falcon de $55 \mathrm{~mL}$.

Para a otimização do processo de digestão, funis de vidro fo- ram acondicionados na extremidade superior dos tubos de digestão, visando favorecer a condensação e o refluxo de gases e vapores gerados na digestão. ${ }^{12}$ Visando anular o efeito da variação espacial da temperatura dentro do bloco digestor, os tubos de digestão contendo as amostras foram distribuídos ao acaso e, à medida que secavam e atingiam o volume desejado (término da digestão), os tubos eram retirados do bloco digestor. Procedimento idêntico foi adotado no método de digestão em que se utilizou a água-régia.

\section{Digestão com água-régia (DAR)}

Conforme descrito no procedimento anterior, foram utilizados 100 e $200 \mathrm{mg}$ de amostra com a adição de $6 \mathrm{~mL}$ de água régia ${ }^{13}$ $\left(\mathrm{HCl}: \mathrm{HNO}_{3}, 3: 1, \mathrm{v} / \mathrm{v}\right)$, que foi adicionada na noite anterior à digestão. A amostra permaneceu em repouso por $16 \mathrm{~h}$, visando a prédigestão. A seguir, iniciou-se a digestão no bloco com a elevação gradual da temperatura até $180{ }^{\circ} \mathrm{C}$. A amostra foi digerida até restar cerca de $1 \mathrm{~mL}$ de ácido, seguindo-se marcha similar à da digestão nítrico-perclórica. Quando não houve clareamento do extrato, adicionaram-se mais $4 \mathrm{~mL}$ da mistura digestora e procedeu-se a digestão até a redução de volume do ácido $( \pm 1 \mathrm{~mL})$. Posteriormente, as amostras foram retiradas do bloco, resfriadas em temperatura ambiente e o extrato digerido teve seu volume completado para $10 \mathrm{~mL}$ com água bidestilada. Em sequiência, o extrato foi filtrado com membrana celulósica com $0,45 \mu \mathrm{m}$ de diâmetro e transferido para frasco tipo Falcon de $55 \mathrm{~mL}$.

\section{Digestão em forno de microondas (DMIC)}

As digestões assistidas por radiação microondas foram realizadas com o emprego de forno microondas. As amostras foram pesadas (100 e $200 \mathrm{mg}$ ) e colocadas nos frascos. Em seguida, adicionaram-se $10 \mathrm{~mL}$ de $\mathrm{HNO}_{3}$ p.a. e os frascos foram fechados e colocados para aquecer durante 10 min (protocolo EPA 3051). No extrato obtido, adicionaram-se mais $10 \mathrm{~mL}$ de água bidestilada, sendo posteriormente filtrado para se proceder à análise multielementar, baseado na metodologia descrita pela agência de proteção ambiental dos Estados Unidos. ${ }^{14}$

\section{Digestão em forno tipo mufla (DMU)}

$\mathrm{O}$ último procedimento de digestão foi baseado na metodologia descrita pela AOAC, ${ }^{15}$ com modificações. Utilizou-se um forno tipo mufla, pesaram-se 100 e $200 \mathrm{mg}$ de amostra, que foram acondicionadas em cadinhos de porcelana e, em seguida, colocadas no forno; a temperatura foi elevada gradualmente até $550{ }^{\circ} \mathrm{C}$ e mantida nesse nível por $2 \mathrm{~h}$. Após esse tempo, a cinza residual foi dissolvida em $5 \mathrm{~mL}$ de $\mathrm{HNO}_{3}$ p.a. concentrado e o extrato obtido foi dilu-

Tabela 1. Caracterização dos resíduos orgânicos estudados

\begin{tabular}{|c|c|c|c|c|c|c|c|}
\hline Material orgânico & $\mathrm{pH}^{(1)}$ & $\begin{array}{l}\mathrm{CE}^{(2)} \\
\mathrm{dS} \mathrm{m}^{-1}\end{array}$ & $\mathrm{MO}^{(3)}$ & $\begin{array}{c}\mathrm{COT}^{(4)} \\
\%\end{array}$ & N-total & \multicolumn{2}{|c|}{$\mathrm{g} \mathrm{kg}^{-1}$} \\
\hline Esterco suíno & $6,5 \pm 0,1$ & $0,7 \pm 0,02$ & $39,5 \pm 0,2$ & $22,8 \pm 0,8$ & $1,9 \pm 0,1$ & $3,9 \pm 0,5$ & $6,0 \pm 0,9$ \\
\hline Esterco bovino & $8,0 \pm 0,1$ & $3,2 \pm 0,12$ & $25,5 \pm 0,1$ & $12,2 \pm 1,1$ & $1,1 \pm 0,1$ & $4,7 \pm 0,3$ & $9,0 \pm 0,5$ \\
\hline Esterco galinha & $7,4 \pm 0,1$ & $9,5 \pm 0,02$ & $45,3 \pm 0,3$ & $19,2 \pm 0,7$ & $4,6 \pm 0,1$ & $11,5 \pm 0,4$ & $35,7 \pm 0,3$ \\
\hline Esterco codorna & $7,9 \pm 0,1$ & $11,6 \pm 0,10$ & $46,6 \pm 0,7$ & $22,7 \pm 1,4$ & $3,3 \pm 0,2$ & $15,3 \pm 1,2$ & $28,3 \pm 0,6$ \\
\hline Composto & $8,5 \pm 0,1$ & $5,8 \pm 0,04$ & $24,5 \pm 0,2$ & $13,6 \pm 0,8$ & $1,2 \pm 0,1$ & $2,5 \pm 0,5$ & $3,7 \pm 0,4$ \\
\hline Material húmico & $4,2 \pm 0,1$ & $5,0 \pm 0,10$ & $43,5 \pm 0,1$ & $18,4 \pm 0,3$ & $1,1 \pm 0,1$ & $71,5 \pm 2,3$ & $4,9 \pm 0,5$ \\
\hline Substrato orgânico & $5,4 \pm 0,1$ & $3,9 \pm 0,04$ & $62,8 \pm 1,3$ & $31,3 \pm 0,4$ & $0,6 \pm 0,1$ & $2,3 \pm 0,4$ & $6,9 \pm 0,6$ \\
\hline Lodo de esgoto 1 & $3,3 \pm 0,1$ & $3,9 \pm 0,04$ & $44,5 \pm 1,1$ & $21,7 \pm 0,6$ & $2,5 \pm 0,1$ & $22,3 \pm 0,5$ & $25,9 \pm 0,4$ \\
\hline Lodo de esgoto 2 & $7,3 \pm 0,1$ & $5,1 \pm 0,10$ & $11,3 \pm 0,2$ & $2,3 \pm 0,3$ & $0,8 \pm 0,1$ & $0,2 \pm 0,0$ & $3,1 \pm 0,4$ \\
\hline
\end{tabular}

${ }^{(1)} \mathrm{Ca}(\mathrm{Cl})_{2}$ 0,01 mol L-1; ${ }^{(2)} \mathrm{CE}$ - Condutividade Elétrica; ${ }^{(3)} \mathrm{MO}$ - Matéria orgânica; ${ }^{(4)} \mathrm{COT}$ - Carbono orgânico total; ${ }^{(5)} \mathrm{C}-\mathrm{FAH}$ - carbono fração ácido húmico; (6) C-FAF - carbono fração ácido fúlvico. \pm - Desvio-padrão da média. 
ído para $20 \mathrm{~mL}$ com água bidestilada e filtrado com membrana celulósica com $0,45 \mu \mathrm{m}$ de diâmetro, para posterior análise multielementar.

\section{Análise multielementar}

Após a etapa de digestão, os extratos foram submetidos à análise multielementar para determinação dos teores de $\mathrm{Ca}, \mathrm{Mg}, \mathrm{Cu}$, Mn e Zn em espectrômetro de absorção atômica de chama aracetileno e os comprimentos de onda utilizados foram 422,7; 285,2; 324,$8 ; 279,5$ e $213,9 \mathrm{~nm}$, respectivamente. Nesse mesmo extrato, foram determinados os teores de S por turbidimetria, ${ }^{16}$ que se baseia na turbidez formada pela precipitação de sulfato pelo cloreto de bário e leitura em espectrofotômetro a $420 \mathrm{~nm}$, de $\mathrm{P}$ por colorimetria, ${ }^{17}$ pela formação do complexo fosfoantimoniomolíbdico de cor azul e leitura em espectrofotômetro a $660 \mathrm{~nm}$ e de $\mathrm{K}$ por fotometria de chama. Em todos os procedimentos de digestão realizados, foram empregadas amostras certificadas de folhas de tomate do National Institute of Standards and Technology (NIST) SRM 1573a. Essas amostras foram escolhidas por conterem valores conhecidos de teores de elementos químicos, que foram determinados nos resíduos analisados. Em todos os procedimentos de digestão utilizaram-se amostras em branco, as quais foram lidas e os teores determinados foram considerados nos cálculos para a determinação do teor final dos elementos avaliados. No preparo das soluções para as curvas-padrão utilizaram-se sempre as mesmas soluções ácidas empregadas em cada procedimento, para evitar interferências advindas da matriz ácida.

\section{Reagentes e instrumentação}

Para os procedimentos de digestão dos resíduos utilizou-se ácido nítrico, ácido clorídrico e ácido perclórico da Quimex ${ }^{\circledR}$. No preparo das soluções para as curvas-padrão utilizaram-se reagentes com pureza analítica da Merck $^{\circledR}$ e em todos os procedimentos utilizouse água desmineralizada e bidestilada (Bi-destilador marca Tecnal, modelo TE 178/5).

O bloco digestor utilizado foi da marca Tecnal ${ }^{\circledR}$, modelo TE 040/25 com capacidade para 40 provas, com corpo de alumínio e termostato. O forno de microondas foi da marca CEM, modelo Mars 5 com frasco de alta pressão de polietileno modificado (TFM) com volume de $50 \mathrm{~mL}$. As condições de operação foram: pressão de $0,76 \mathrm{MPa}$ e temperatura de aproximadamente $175^{\circ} \mathrm{C}$. O forno tipo mufla utilizado foi da marca Lavoisier Modelo $400 \mathrm{D}$ e operou a uma temperatura máxima de $550{ }^{\circ} \mathrm{C}$.

Para determinação dos teores de elementos químicos, utilizou-se aparelho de absorção atômica de chama ar-acetileno, Perkin Elmer Modelo AAnalyst 800, espectrofotômetro Micronal, modelo B442 e fotômetro de chama, Digimed modelo DM-61

\section{Análise estatística}

Os dados obtidos foram submetidos à análise de variância, para se avaliar os métodos/técnicas mais eficientes na digestão e caracterização química dos resíduos orgânicos. Foi utilizado o delineamento inteiramente casualizado, com quatro repetições. Foram considerados como fatores de estudo as massas de amostras e os métodos de digestão. Realizou-se a análise de variância pelo teste de $\mathrm{F}$ e, quando houve significância $(\mathrm{p}<0,05)$, as médias foram comparadas pelo teste de Tukey, a 5\% de probabilidade, por meio do programa estatístico Sisvar. ${ }^{18}$ No caso de variação significativa entre os fatores, realizou-se o desdobramento da mesma.

\section{RESULTADOS E DISCUSSÃO}

Em geral, os índices de recuperação de nutrientes foram bastante variáveis para os diferentes resíduos. Isso deve estar relacionado às diferentes características dos resíduos, que possuem teores variáveis de carbono orgânico total (COT) e diferentes graus de humificação, como pode ser observado pelos teores de C-substâncias húmicas, representados pelo carbono na fração ácido húmico (C-FAH) e carbono na fração ácido fúlvico (C-FAF) (Tabela 1), o que implica em diferentes graus de resistência à digestão da matriz orgânica. Além disso, os índices variáveis são consequiências da capacidade de extração de cada método empregado. Assim, em todos esses métodos, a recuperação somente se aproxima do total, dado que esses métodos não são capazes de dissolver silicatos, mas são eficientes para solubilizar metais ligados aos constituintes orgânicos. ${ }^{3}$ Para a dissolução total, é necessário o uso de ácido fluorídrico (HF) combinado com ácidos fortes, entretanto, o uso de $\mathrm{HF}$ em rotinas de laboratório não é recomendado, devido aos riscos de acidentes, por se tratar de ácido altamente corrosivo e que requer cuidados no manuseio. ${ }^{19}$

Os extratos obtidos pela digestão nítrico-perclórica (DNP) apresentaram coloração transparente, sem sinais visíveis de material residual, para todos os resíduos estudados. Em alguns casos, como na digestão do material húmico, substrato orgânico e composto orgânico, foi necessário adicionar mais $4 \mathrm{~mL}$ de ácido nítrico, além dos 6 $\mathrm{mL}$ iniciais, para uma digestão completa da matriz orgânica. Já na digestão com água-régia (DAR), os extratos obtidos apresentaram uma coloração amarelada, provavelmente devido à presença de óxidos nitrosos. Também foi possível perceber a presença de resíduos sólidos no fundo dos tubos de digestão. Isso indica que não houve uma abertura completa da matriz orgânica por esse método, mesmo colocando-se $4 \mathrm{~mL}$ adicionais aos $6 \mathrm{~mL}$ iniciais. Os extratos finais obtidos na digestão em forno de microondas (DMIC) apresentaram coloração amarela e, alguns extratos, sobretudo aqueles de materiais mais recalcitrantes (material húmico, substrato orgânico e composto orgânico) apresentaram coloração escura, impossibilitando a determinação do $\mathrm{S}$ por meio do método turbidimétrico.

Os resultados obtidos para a amostra padrão (certificada) estão de acordo com aqueles obtidos para os resíduos, ou seja, os métodos cuja recuperação foi maior para os elementos químicos avaliados também apresentaram maior recuperação nessa amostra, conforme mostrado na Figura 1. O gráfico foi obtido a partir dos valores de recuperação desses elementos químicos na amostra padrão, apresentados na Tabelas 2 e na Tabela $1 \mathrm{~S}$ (material suplementar). O valor de $100 \%$ corresponde aos teores certificados de elementos químicos.

Os teores de macronutrientes nos diversos resíduos orgânicos, obtidos pelos quatro métodos de digestão, nas duas massas de amostras, são apresentados na Tabela 2. Em todos os casos, houve variação significativa entre métodos de digestão e massa de amostra digerida.

Os resultados indicam que a maior recuperação de $\mathrm{P}$ foi obtida pelo método DMIC para o esterco de suíno, pela DAR no esterco de bovino e pela DNP no esterco de galinha e material húmico, na massa de $100 \mathrm{mg}$. Para os demais resíduos, a DNP, na massa de $200 \mathrm{mg}$, foi o método com melhor recuperação de P. Para K, o método DMIC proporcionou maior recuperação, nas duas massas de amostras, nos estercos de galinha e de codorna e substrato orgânico. Para os estercos de suíno e bovino, a DMU propiciou a obtenção de maiores teores de nutrientes nas duas massas. No material húmico e lodos de esgoto 1 e 2, a DAR recuperou maiores teores de $\mathrm{K}$ nas massas de 100 e $200 \mathrm{mg}$, tendo, nos dois primeiros, a DNP, na massa de 200 $\mathrm{mg}$, também obtido um índice satisfatório de recuperação.

Nas Figuras $1 \mathrm{~S}$ e $2 \mathrm{~S}$ encontram-se as porcentagens de recuperação relativa dos teores de macro e micronutrientes avaliados nos 
Tabela 2. Teores de macronutrientes obtidos por quatro métodos de digestão e duas massas de amostras em resíduos orgânicos de origens diversas

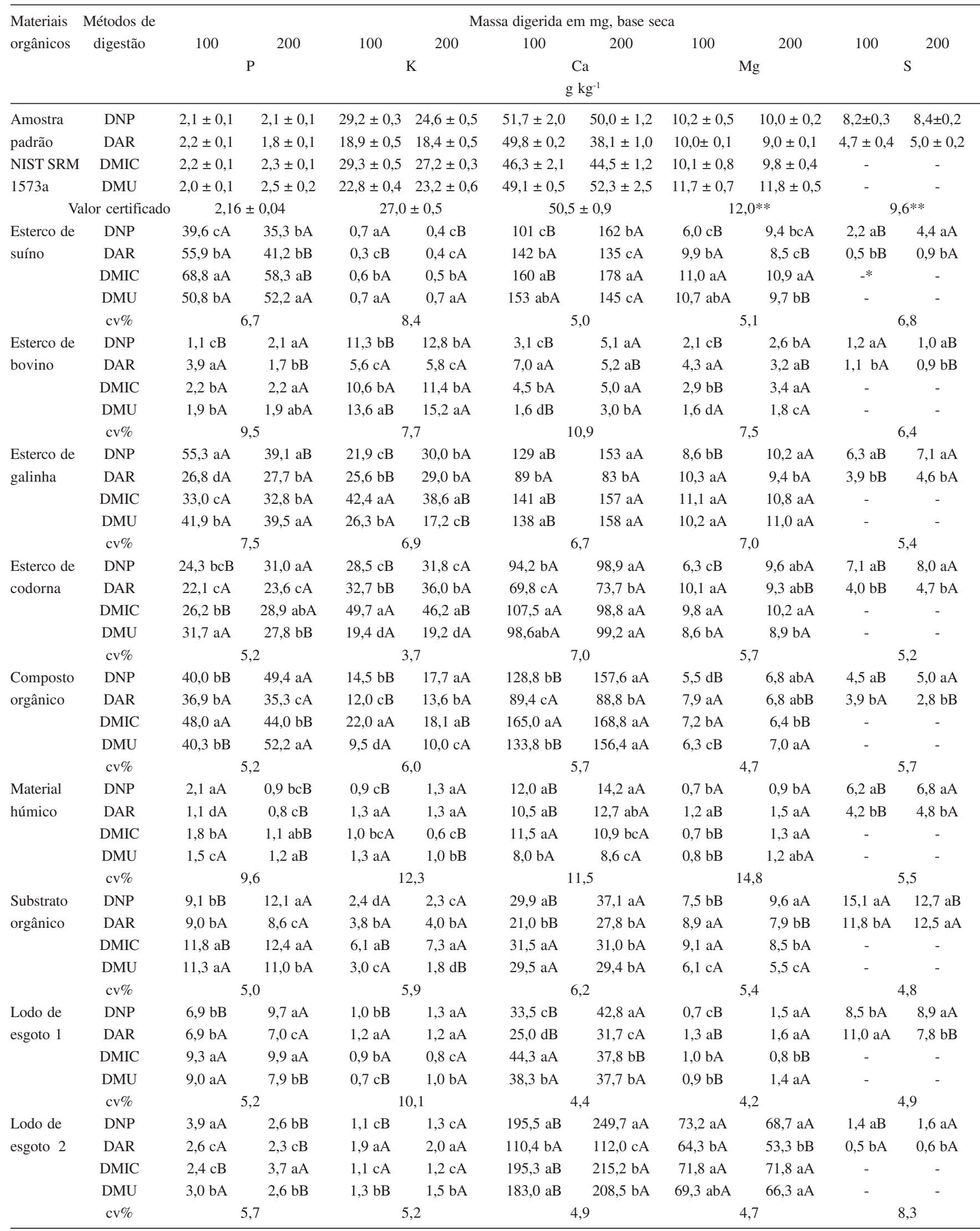

Médias seguidas por letras minúsculas iguais na coluna e maiúsculas iguais na linha não diferem entre si, pelo teste de Tukey (p $\leq 0,05$ ). \pm - Desvio-padrão da média. - = valor não determinado** = valores de referência não certificados. DNP - Digestão nítrico-perclórica; DAR - Digestão com água-régia; DMIC Digestão em forno microondas (EPA 3051); DMU - Digestão em forno tipo mufla. 
Padrão NIST SRM 1573a

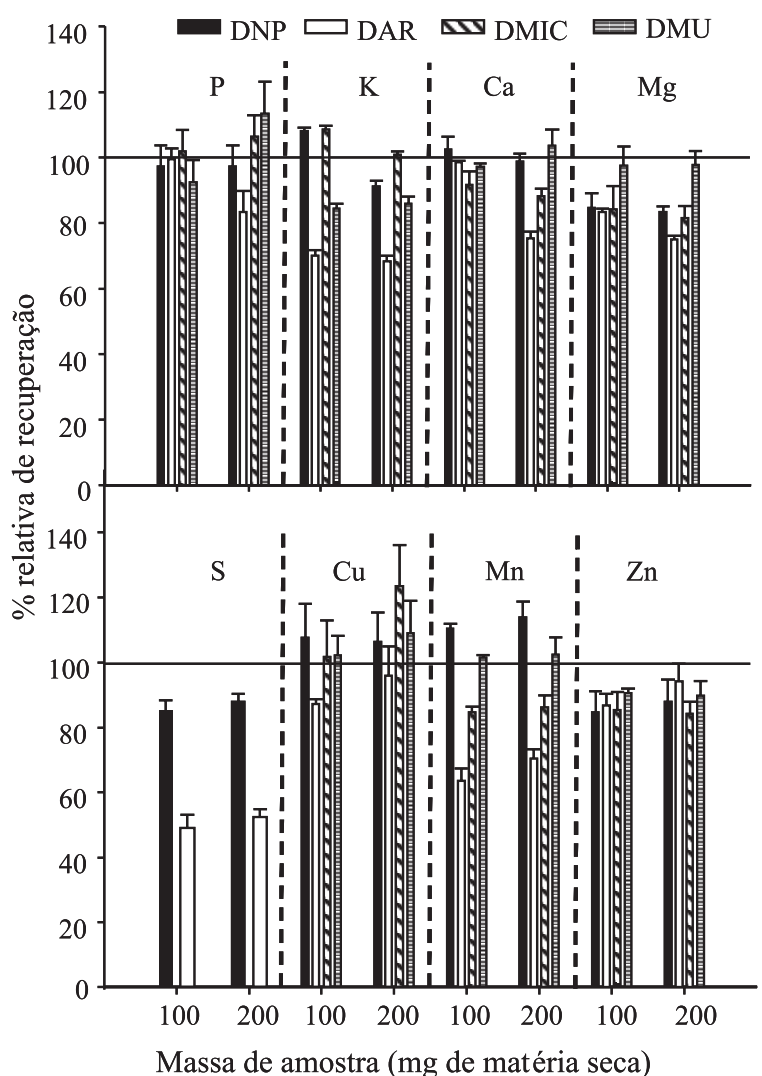

Figura 1. Porcentagem relativa de recuperação dos teores de macro e micronutrientes obtidos por quatro métodos de digestão (DNP - digestão nítrico-perclórica; DAR - digestão com água-régia; DMIC - digestão em forno microondas; DMU - digestão em forno tipo mufla) e duas massas de amostras (100 e $200 \mathrm{mg}$ ) em amostras certificadas de folha de tomate (Padrão NIST SRM 1573a)

diversos resíduos orgânicos. Nesse caso, adotou-se o seguinte critério: o maior teor recuperado de cada elemento químico (Tabelas 2), nos quatro métodos de digestão e nas duas massas de amostras, foi considerado como $100 \%$ de recuperação e os demais valores foram normalisados. Durante a discussão dos resultados, foi (foram) considerado(s) como de maior eficiência o(s) método(s) com maior capacidade de recuperação.

Em geral, para Ca e Mg, o método DNP na massa de $200 \mathrm{mg}$ foi o método com melhor desempenho, com exceção do esterco de bovino, em que o método DAR, na massa de $100 \mathrm{mg}$, obteve um desempenho superior aos demais métodos (Figura 1S). Sahrawat et al. ${ }^{20}$ compararam o procedimento de digestão seca (mufla) com a digestão triácida $\left(\mathrm{HNO}_{3}: \mathrm{H}_{2} \mathrm{SO}_{4}: \mathrm{HClO}_{4}, 9: 2: 1, \mathrm{v} / \mathrm{v}\right)$ em amostras de sorgo e arroz e concluíram que a digestão seca recuperou maiores teores de $\mathrm{Ca}$ e deve ser preferida à digestão triácida. Também concluíram que ambos os procedimentos se mostraram satisfatórios para a determinação de $\mathrm{K}, \mathrm{Mg}, \mathrm{Mn}$ e $\mathrm{Zn}$.

Para a determinação do $\mathrm{S}$, de maneira geral, a DNP, associada à massa de $200 \mathrm{mg}$, obteve melhor desempenho na maioria dos resíduos, à exceção apenas do substrato orgânico e do esterco de bovino, nos quais a massa $100 \mathrm{mg}$ propiciou maior recuperação de $\mathrm{S}$.

Para o lodo de esgoto 1, a DAR, na massa de $100 \mathrm{mg}$, foi o método que mais recuperou $\mathrm{S}$. $\mathrm{O}$ enxofre não pôde ser determinado pelo método DMU e a hipótese para explicar esse fato é que, durante a incineração em alta temperatura (cerca de $550{ }^{\circ} \mathrm{C}$ ), o $\mathrm{S}$ foi perdido por volatilização em grandes quantidades, impedindo sua determinação por turbidimetria. Segundo Miyazawa et al.,$^{21}$ em amostras de tecido vegetal, a volatilização de $\mathrm{S}$ se inicia em temperaturas superiores a $100{ }^{\circ} \mathrm{C}$ e a digestão seca sob temperaturas elevadas não deve ser recomendada para a determinação desse elemento.

Os teores de micronutrientes $\mathrm{Cu}, \mathrm{Mn}$ e $\mathrm{Zn}$, obtidos pelos quatro métodos de digestão e duas massas de amostras, são apresentados na Tabela 1S. Houve variação significativa entre métodos de digestão e massa de amostra digerida. Na recuperação de $\mathrm{Cu}, \mathrm{Mn}$ e $\mathrm{Zn}$, em geral, a DNP, na massa de $200 \mathrm{mg}$, foi o método mais estável, ou seja, o que obteve os melhores resultados para a maioria dos resíduos orgânicos avaliados neste estudo (Figuras $1 \mathrm{~S}$ e 2S).

Apenas para o esterco de bovino, a DMIC, utilizando $100 \mathrm{mg}$ de amostra e no lodo de esgoto 2 e a DMU, na massa de $200 \mathrm{mg}$, apresentaram resultados superiores na recuperação de $\mathrm{Cu}$. Sahrawat et al. ${ }^{20}$ verificaram que a digestão pela mufla em amostras de sorgo obteve resultados mais confiáveis que a digestão triácida para a determinação de $\mathrm{Cu}$. Abreu et al. ${ }^{22}$ obtiveram maiores teores de metais pesados, incluindo $\mathrm{Cu}, \mathrm{Mn}$ e $\mathrm{Zn}$, em amostra de esterco de suíno, lodo de esgoto e composto de lixo, utilizando água-régia em digestão com microondas, comparativamente à digestão com ácido nítrico em microondas e nítrico-perclórica em bloco digestor. De acordo com esse resultado, pode-se afirmar que a água-régia em aquecimento com microondas pode apresentar uma maior eficiência de recuperação que no aquecimento pelo bloco. Para alguns materiais estudados (substrato orgânico e lodos de esgoto 1 e 2), a DMU recuperou os menores teores de Mn, nas massas de 100 e $200 \mathrm{mg}$. Zheljazkov e Warman ${ }^{3}$ afirmaram que a recuperação dos elementos é influenciada não só pelo método empregado, mas também pela natureza do composto orgânico e que a digestão pela mufla não é um procedimento eficiente para a recuperação de elementos em compostos.

\section{CONCLUSÕES}

A digestão nítrico-perclórica, quando se utiliza a massa de 200 mg de amostra, é a que propicia a recuperação dos maiores teores de nutrientes, para a maioria dos resíduos orgânicos estudados, comparativamente aos outros procedimentos avaliados.

O método de digestão nítrico-perclórica apresenta baixa recuperação de $\mathrm{K}$ nas duas massas de amostras (100 e $200 \mathrm{mg}$ ), em relação aos demais métodos, provavelmente devido à baixa solubilidade do perclorato de potássio.

O método de digestão utilizando a mufla, por causar a volatilização de $\mathrm{S}$ e o método do forno de microondas (EPA 3051), por produzir extratos de coloração escura, não se mostram adequados para a determinação por turbidimetria do teor de $\mathrm{S}$ presente nos resíduos orgânicos analisados.

Em geral, o método de digestão que utiliza a água-régia é o que propicia menor índice de recuperação dos elementos químicos avaliados nos resíduos orgânicos.

\section{MATERIAL SUPLEMENTAR}

Em http://quimicanova.sbq.org.br encontram-se disponíveis a Figura 1S, que apresenta a porcentagem relativa de recuperação dos teores de macro e micronutrientes obtidos por quatro métodos de digestão, nas massas digeridas: $1(100 \mathrm{mg})$ e $2(200 \mathrm{mg})$, em amostras de esterco de suíno, bovino, galinha e codorna, e a Figura $2 \mathrm{~S}$ que apresenta a porcentagem relativa de recuperação dos teores de macro e micronutrientes obtidos por quatro métodos de digestão, nas massas digeridas: 1 (100 mg) e $2(200 \mathrm{mg})$, em amostras de composto orgânico, material húmico, substrato orgânico e lodo 
de esgoto 1. Também encontra-se disponível a Tabela $1 \mathrm{~S}$ com os teores de micronutrientes $(\mathrm{Cu}, \mathrm{Mn}$ e $\mathrm{Zn}$ ) obtidos por quatro métodos de digestão, nas massas digeridas 100 e 200 mg, em resíduos orgânicos de origens diversas.

\section{AGRADECIMENTOS}

Ao apoio financeiro da Fundação da Amparo à Pesquisa do Estado de Minas Gerais (Processo CAG 1776/06) e ao Prof. J. J. G. S. M. Marques pela orientação e pela cessão do laboratório de Pedologia e Geoquímica Ambiental para a realização de parte das análises.

\section{REFERÊNCIAS}

1. Hseu, Z. Y.; Bioresour. Technol. 2004, 95, 53.

2. Rodushkin, I.; Ruth, T.; Huhtasaari, A.; Anal. Chim. Acta 1999, 378, 191.

3. Zheljazkov, V. D.; Warman, P. R.; Compost Science and Utilization 2002, 10,197 .

4. Azcue, J. ; Mudroch, A.; Int. J. Environ. Anal. Chem. 1994, 12, 211.

5. Sastre, J.; Sahuquillo, A.; Vidal, M.; Rauret, G.; Anal. Chim. Acta 2002, $462,59$.

6. Ministério da Agricultura, Brasil; Decomposição da matéria orgânica de compostos de lixo urbano e posterior preparo de extratos nítrico-perclórico, Campinas: São Paulo, 2001.

7. Egreja Filho, F. B.; Dissertação de Mestrado, Universidade Federal de Viçosa, Brasil, 1993.

8. Boeira, R. C.; Maximiliano, V. C. B.; Fay, E. F.; Souza, M. D.; Moraes,
M. P. G.; Resumos do Congresso Brasileiro de Ciência e Tecnologia em Resíduos e Desenvolvimento sustentável, Florianópolis, Brasil, 2004.

9. Alkanani, T.; Friel, J. K.; Jackson, S. E.; Longerich, H. P.; J. Agric. Food Chem. 1994, 42, 1965.

10. Hseu, Z. Y.; Chen, Z. S.; Tsai, C. C.; Tsui, C. C.; Cheng, S. F.; Liu, C. L.; Lin, H. T.; Water, Air, Soil Pollut. 2002, 141, 189.

11. Moral, R.; Pedreño, J. N.; Gómez, I.; Mataix, J.; Commun. Soil Sci. Plant Anal. 1996, 27, 753

12. Tedesco, M. J.; Gianello, C.; Bissani, C. A.; Bohnen, H.; Volkweiss, S. J.; Análise de solo, plantas e outros materiais, $2^{\mathrm{a}}$ ed., ??: ???, 1995.

13. McGrath, S.; Cunliffe, C. H. J.; J. Sci. Food Agric. 1985, 36, 794.

14. United States Environmental Protection Agency-USEPA; SW-846: test methods for evaluating solid waste, physical and chemical methods, Washington, 1998.

15. Capar, S. G.; Gajan, R. J.; Madzsar, E.; Albert, R. H.; Sanders, M.; Zyren, J.; J. Assoc. Off. Anal. Chem. 1982, 65, 978.

16. Vitti, G. C.; Avaliação e interpretação do enxofre no solo e na planta, ??: Jaboticabal, 1989.

17. Empresa Brasileira de Pesquisa Agropecuária - EMBRAPA; Manual de análises químicas de solos, plantas e fertilizantes, Brasília, 1999.

18. Ferreira, D. F.; Resumos da $45^{a}$ Reunião Anual da Região Brasileira da Sociedade Internacional de Biometria, São Carlos, Brasil, 2000.

19. Hseu, Z. Y.; Chen, Z. S.; Tsai, C. C.; Tsui, C. C.; Cheng, S. F.; Liu, C. L.; Lin, H. T.; Water, Air, Soil Pollut. 2002, 141, 189.

20. Sahrawat, K. L.; Ravi - Kumar, G.; Rao, J. K.; Commun. Soil Sci. Plant Anal. 2002, 33, 95 .

21. Miyazawa, M.; Bianchi, S.; Oliveira, E. L.; Diehl, R. C.; Resumos $27^{a}$ Reunião Brasileira de Fertilidade do Solo e Nutrição de Plantas, Bonito, Brasil, 2006.

22. Abreu, M. F.; Berton, R. S.; Andrade, J. C.; Commun. Soil Sci. Plant Anal. 1996, 27, 1125 . 

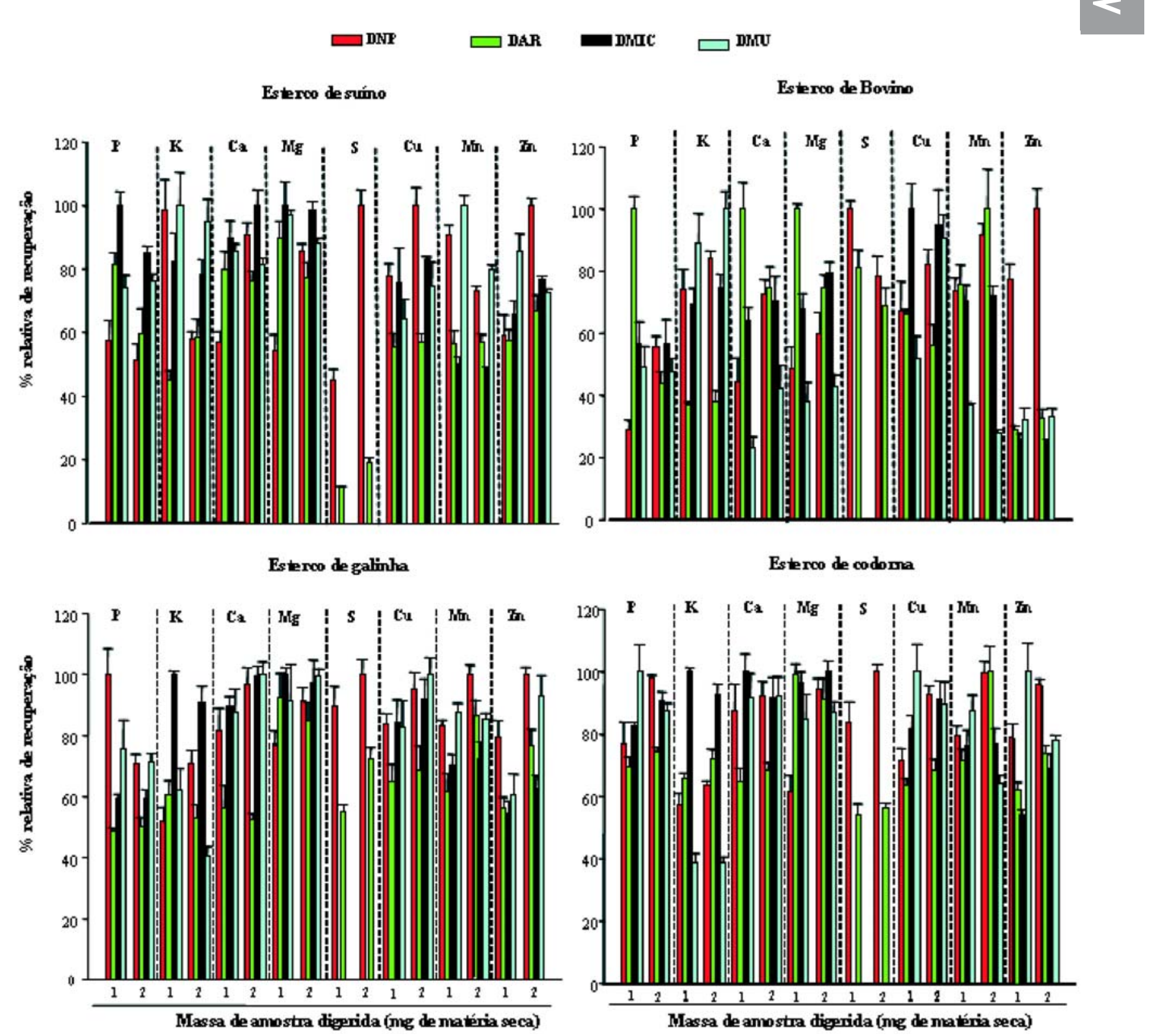

Figura 1S. Porcentagem relativa de recuperação dos teores de macro e micronutrientes obtidos por quatro métodos de digestão (DNP - digestão nítricoperclórica; DAR - digestão com água-régia; DMIC - digestão em forno de microondas (EPA 3051); DMU - digestão em forno tipo mufla) em amostras de esterco de suíno, bovino, galinha e codorna. Massa digerida: 1 (100 mg); 2 (200 mg).

*e-mail: leonidasmelobr@yahoo.com.br 
Tabela 1S. Teores de micronutrientes obtidos por quatro métodos de digestão e duas massas de amostras em resíduos orgânicos de origens diversas

\begin{tabular}{|c|c|c|c|c|c|c|c|}
\hline \multirow{3}{*}{$\begin{array}{l}\text { Resíduo } \\
\text { orgânico }\end{array}$} & \multirow{3}{*}{$\begin{array}{l}\text { Método de } \\
\text { digestão }\end{array}$} & \multicolumn{3}{|c|}{ Massa digerida em mg, base seca } & \multirow[b]{2}{*}{200} & \multirow[b]{2}{*}{100} & \multirow[b]{2}{*}{200} \\
\hline & & 100 & 200 & 100 & & & \\
\hline & & \multicolumn{2}{|c|}{$\mathrm{Cu}$} & \multicolumn{2}{|c|}{$\begin{array}{c}\mathrm{Mn} \\
\mathrm{mg} \mathrm{kg}^{-1}\end{array}$} & \multicolumn{2}{|c|}{$\mathrm{Zn}$} \\
\hline Amostra & DNP & $5,1 \pm 0,4$ & $5,0 \pm 0,4$ & $271 \pm 4$ & $280 \pm 12$ & $26,2 \pm 2,0$ & $27,2 \pm 2,1$ \\
\hline padrão & DAR & $4,1 \pm 0,1$ & $4,5 \pm 0,4$ & $157 \pm 9$ & $173 \pm 7$ & $26,8 \pm 1,1$ & $29,1 \pm 1,7$ \\
\hline NIST SRM & DMIC & $4,8 \pm 0,5$ & $5,8 \pm 0,6$ & $208 \pm 4$ & $212 \pm 9$ & $26,4 \pm 1,7$ & $26,0 \pm 1,2$ \\
\hline \multirow[t]{2}{*}{$1573 \mathrm{a}$} & DMU & $4,8 \pm 0,3$ & $5,1 \pm 0,5$ & $249 \pm 2$ & $252 \pm 13$ & $27,9 \pm 0,5$ & $27,7 \pm 1,5$ \\
\hline & Valor certificado & \multicolumn{2}{|c|}{$4,70 \pm 0,14$} & \multicolumn{2}{|c|}{$246 \pm 8$} & \multicolumn{2}{|c|}{$30,9 \pm 0,7$} \\
\hline \multirow[t]{5}{*}{ Esterco de suíno } & DNP & $664 \mathrm{aB}$ & $851 \mathrm{aA}$ & $2539 \mathrm{bA}$ & $2055 \mathrm{bB}$ & $820 \mathrm{bcB}$ & $1388 \mathrm{aA}$ \\
\hline & DAR & $470 \mathrm{cA}$ & $484 \mathrm{cA}$ & $1578 \mathrm{cA}$ & $1592 \mathrm{cA}$ & $795 \mathrm{cB}$ & $931 \mathrm{cA}$ \\
\hline & DMIC & $644 \mathrm{abA}$ & $705 \mathrm{bA}$ & $1412 \mathrm{dA}$ & $1378 \mathrm{dA}$ & $914 \mathrm{bB}$ & $1064 \mathrm{bA}$ \\
\hline & DMU & $547 \mathrm{bcB}$ & $634 \mathrm{bA}$ & $2802 \mathrm{aA}$ & $2240 \mathrm{aB}$ & $1189 \mathrm{aA}$ & $1005 \mathrm{bcB}$ \\
\hline & $\mathrm{cv} \%$ & \multicolumn{2}{|c|}{8,3} & \multicolumn{2}{|c|}{3,6} & \multicolumn{2}{|c|}{5,7} \\
\hline \multirow[t]{5}{*}{ Esterco de bovino } & DNP & $19,0 \mathrm{bB}$ & $23,2 \mathrm{aA}$ & $275 \mathrm{aB}$ & $340 \mathrm{aA}$ & $145 \mathrm{aB}$ & $188 \mathrm{aA}$ \\
\hline & DAR & $18,7 \mathrm{bA}$ & $15,9 \mathrm{bA}$ & $283 \mathrm{aB}$ & $372 \mathrm{aA}$ & $55 \mathrm{bA}$ & $61 \mathrm{bA}$ \\
\hline & DMIC & $28,2 \mathrm{aA}$ & $26,8 \mathrm{aA}$ & $262 \mathrm{aA}$ & $267 \mathrm{bA}$ & $48 \mathrm{bA}$ & $46 \mathrm{cA}$ \\
\hline & DMU & $14,7 \mathrm{cB}$ & $25,6 \mathrm{aA}$ & $137 \mathrm{bB}$ & $268 \mathrm{bA}$ & $60 \mathrm{bA}$ & $62 \mathrm{bA}$ \\
\hline & $\mathrm{cv} \%$ & \multicolumn{2}{|c|}{10,0} & & & & \\
\hline Esterco de galinha & DNP & $68,3 \mathrm{aB}$ & $77,6 \mathrm{aA}$ & $693 \mathrm{aB}$ & $834 \mathrm{aA}$ & $581 \mathrm{aB}$ & $729 \mathrm{aA}$ \\
\hline & DAR & $52,9 \mathrm{bA}$ & $56,0 \mathrm{bA}$ & $516 \mathrm{cB}$ & $717 \mathrm{bA}$ & $410 \mathrm{bB}$ & $558 \mathrm{bA}$ \\
\hline & DMIC & $68,8 \mathrm{aA}$ & $74,8 \mathrm{aA}$ & $584 \mathrm{bA}$ & $603 \mathrm{cA}$ & $398 \mathrm{bB}$ & $457 \mathrm{cA}$ \\
\hline & DMU & $67,3 \mathrm{aB}$ & $81,5 \mathrm{aA}$ & $730 \mathrm{aA}$ & $711 \mathrm{bA}$ & $442 \mathrm{bB}$ & $676 \mathrm{aA}$ \\
\hline & $\mathrm{cv} \%$ & & & & & & \\
\hline Esterco de codorna & DNP & $71,5 \mathrm{bcB}$ & $92,9 \mathrm{aA}$ & $430 \mathrm{abB}$ & $537 \mathrm{aA}$ & $924 \mathrm{bB}$ & $1122 \mathrm{aA}$ \\
\hline & DAR & $63,8 \mathrm{cA}$ & $68,3 \mathrm{bA}$ & $385 \mathrm{bB}$ & $539 \mathrm{aA}$ & $729 \mathrm{cB}$ & $861 \mathrm{bcA}$ \\
\hline & DMIC & 81,6 bB & $91,2 \mathrm{aA}$ & $412 \mathrm{bA}$ & $415 \mathrm{bA}$ & $631 \mathrm{cB}$ & $808 \mathrm{cA}$ \\
\hline & DMU & $100,0 \mathrm{aA}$ & $89,5 \mathrm{aB}$ & $471 \mathrm{aA}$ & $346 \mathrm{bB}$ & $1171 \mathrm{aA}$ & 914 bB \\
\hline & $\mathrm{cv} \%$ & & & & & & \\
\hline Composto orgânico & DNP & $96,0 \mathrm{aA}$ & $97,5 \mathrm{aA}$ & $783 \mathrm{bB}$ & $985 \mathrm{aA}$ & $335 \mathrm{aB}$ & $400 \mathrm{aA}$ \\
\hline & DAR & $76,9 \mathrm{bA}$ & $77,1 \mathrm{bA}$ & $943 \mathrm{aA}$ & $908 \mathrm{bA}$ & $262 \mathrm{bB}$ & $285 \mathrm{bA}$ \\
\hline & DMIC & $82,8 \mathrm{bB}$ & $107,5 \mathrm{aA}$ & $576 \mathrm{dA}$ & $554 \mathrm{cA}$ & $169 \mathrm{cB}$ & $196 \mathrm{cA}$ \\
\hline & DMU & $75,2 \mathrm{bB}$ & $100,6 \mathrm{aA}$ & $632 \mathrm{cA}$ & $521 \mathrm{cB}$ & $170 \mathrm{cB}$ & $130 \mathrm{dA}$ \\
\hline & $\mathrm{cv} \%$ & & & & & & \\
\hline Material húmico & DNP & $40,3 \mathrm{aA}$ & $42,8 \mathrm{aA}$ & $45,4 \mathrm{aB}$ & 50,6 aA & $26,4 \mathrm{aA}$ & $22,4 \mathrm{abA}$ \\
\hline & DAR & $34,7 \mathrm{bA}$ & $28,7 \mathrm{cB}$ & $45,9 \mathrm{aA}$ & $41,4 \mathrm{bB}$ & $28,6 \mathrm{aA}$ & $25,0 \mathrm{aA}$ \\
\hline & DMIC & $35,0 \mathrm{bB}$ & $40,0 \mathrm{abA}$ & $37,1 \mathrm{bA}$ & $26,7 \mathrm{cB}$ & $15,1 \mathrm{bB}$ & $19,0 \mathrm{bcA}$ \\
\hline & DMU & $34,3 \mathrm{bA}$ & $35,5 \mathrm{bA}$ & $40,2 \mathrm{bA}$ & $38,7 \mathrm{bA}$ & $23,1 \mathrm{aA}$ & $16,7 \mathrm{cB}$ \\
\hline & $\mathrm{cv} \%$ & & & & & & \\
\hline Substrato orgânico & DNP & $65,1 \mathrm{aA}$ & $69,7 \mathrm{aA}$ & $408 \mathrm{aB}$ & $569 \mathrm{aA}$ & $346 \mathrm{aB}$ & $403 \mathrm{aA}$ \\
\hline & DAR & $44,1 \mathrm{bA}$ & $46,9 \mathrm{bA}$ & $328 \mathrm{bB}$ & $468 \mathrm{bA}$ & $203 \mathrm{bcB}$ & 294 bA \\
\hline & DMIC & $59,0 \mathrm{aB}$ & $72,6 \mathrm{aA}$ & $332 \mathrm{bA}$ & $335 \mathrm{cA}$ & $177 \mathrm{cB}$ & $203 \mathrm{cA}$ \\
\hline & DMU & $30,4 \mathrm{cB}$ & $43,5 \mathrm{bA}$ & $277 \mathrm{cA}$ & $193 \mathrm{~dB}$ & $218 \mathrm{bA}$ & $181 \mathrm{~dB}$ \\
\hline & $\mathrm{cv} \%$ & & & & & & \\
\hline Lodo de esgoto 1 & DNP & $562 \mathrm{aA}$ & $589 \mathrm{aA}$ & $299 \mathrm{aB}$ & $415 \mathrm{aA}$ & $741 \mathrm{aB}$ & $938 \mathrm{aA}$ \\
\hline & DAR & $414 \mathrm{bB}$ & $461 \mathrm{bA}$ & $238 \mathrm{cB}$ & $251 \mathrm{cA}$ & $549 \mathrm{bB}$ & 682 bA \\
\hline & DMIC & $547 \mathrm{aA}$ & $573 \mathrm{aA}$ & $282 \mathrm{bA}$ & $288 \mathrm{bA}$ & $545 \mathrm{bB}$ & $624 \mathrm{cA}$ \\
\hline & DMU & $103 \mathrm{cB}$ & $181 \mathrm{cA}$ & $168 \mathrm{~dB}$ & $181 \mathrm{dA}$ & $300 \mathrm{cA}$ & $269 \mathrm{dA}$ \\
\hline & $\mathrm{cv} \%$ & & & & & & \\
\hline Lodo de esgoto 2 & DNP & $19,0 \mathrm{bcA}$ & $19,8 \mathrm{cA}$ & $440 \mathrm{aB}$ & $505 \mathrm{aA}$ & $120 \mathrm{aB}$ & $137 \mathrm{aA}$ \\
\hline & DAR & 14,6 bA & $13,1 \mathrm{dA}$ & $316 \mathrm{bcB}$ & $497 \mathrm{aA}$ & $106 \mathrm{bA}$ & $107 \mathrm{bA}$ \\
\hline & DMIC & $23,0 \mathrm{bB}$ & $30,6 \mathrm{bA}$ & $345 \mathrm{bA}$ & $342 \mathrm{bA}$ & $87 \mathrm{cB}$ & $100 \mathrm{bA}$ \\
\hline & DMU & $29,7 \mathrm{aB}$ & $43,7 \mathrm{aA}$ & $290 \mathrm{cA}$ & $259 \mathrm{cB}$ & $57 \mathrm{dA}$ & $55 \mathrm{cA}$ \\
\hline & $\mathrm{cv} \%$ & & & & & & \\
\hline
\end{tabular}

Médias seguidas por letras, minúsculas, iguais na coluna e, maiúsculas, iguais na linha não diferem entre si, pelo teste de Tukey (p $\leq 0,05$ ). \pm - Desvio-padrão da média. DNP - Digestão nítrico-perclórica; DAR - Digestão com água-régia; DMIC - Digestão em forno de microondas (EPA 3051); DMU - Digestão em forno tipo mufla. 

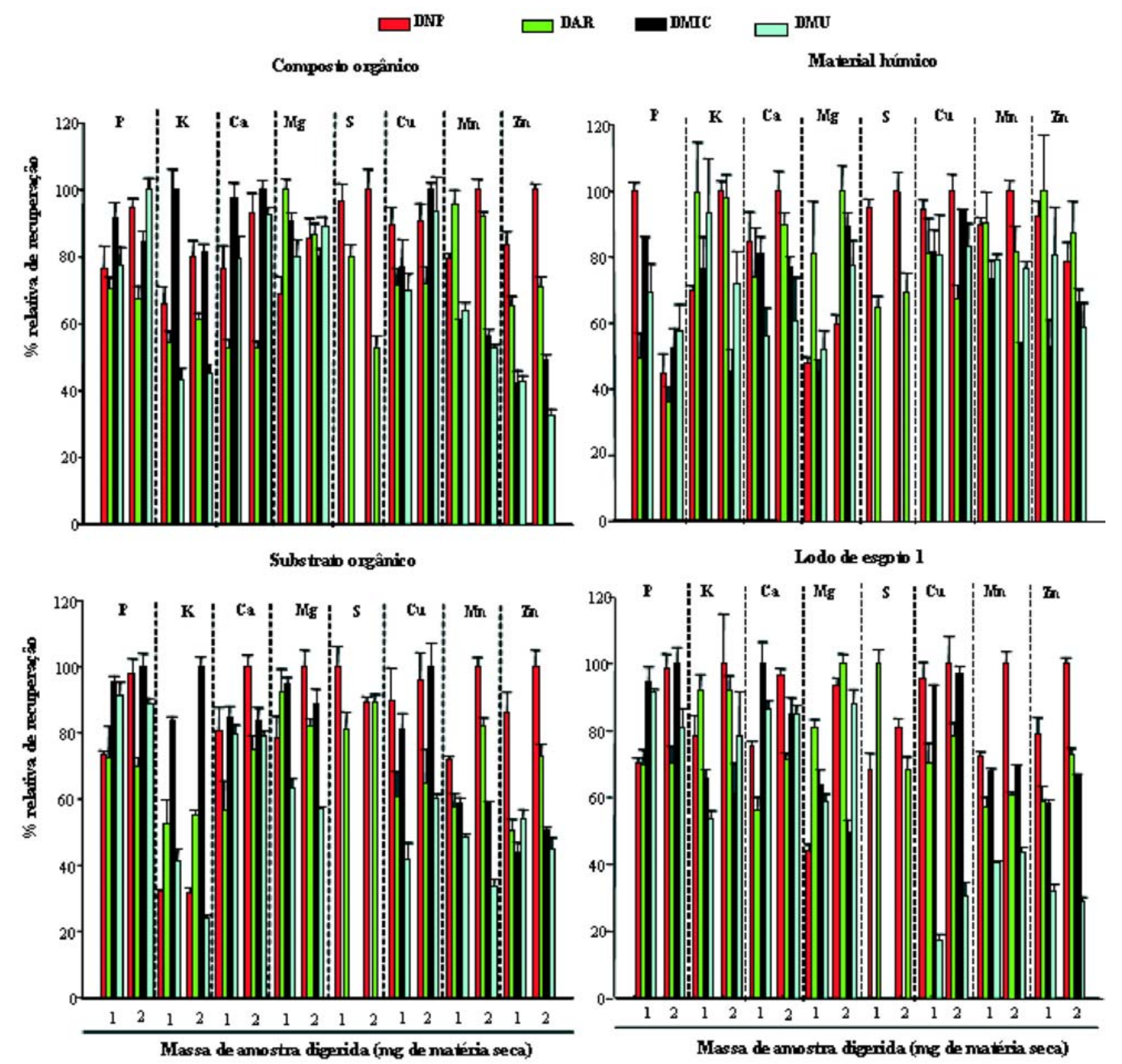

Figura 2S. Porcentagem relativa de recuperação dos teores de macro e micronutrientes obtidos por quatro métodos de digestão (DNP - digestão nítricoperclórica; DAR - digestão com água-régia; DMIC - digestão em forno de microondas (EPA 3051); DMU - digestão em forno tipo mufla) em amostras de composto orgânico, material húmico, substrato orgânico e lodo de esgoto. Massa digerida: 1 (100 mg); 2 (200 mg). 